\title{
ULTRASTRUCTURE ANALYSIS OF PRIMARY AND PERMANENT TEETH WITH GENERALIZED AMELOGENESIS IMPERFECTA; IN-VITRO STUDY
}

\author{
Walid El-Sayed **** and Ghada Mohammed Said*
}

\begin{abstract}
Amelogenesis or enamel formation is a biomineralization process in which specialized epithelial derived cells, ameloblasts, secrete extracellular matrix which later will be mineralized. amelogenesis can be divided into two main stages: the secretory stage and the maturation stage. Objectives: This work aims to investigate the ultrastructure phenotype of permanent and deciduous teeth with generalized enamel defects.
\end{abstract}

Material and Methods; Teeth whether deciduous or permanent (exfoliated or extracted for clinical reasons), were collected from affected individuals. Scanning Electron Microscopy Energy Dispersive X-Ray Spectroscopy techniques were applied. Enzyme digestions was done by using either $\alpha$ - chymotrypsin (Sigma-UK) or lipase (Sigma-UK).

Results: Examination of sections of affected teeth using Scanning Electron Microscopy showed abnormal enamel rods that were covered by amorphous material. This pattern was consistent of both primary and secondary dentitions. Energy Dispersive X-Ray Spectroscopy showed increase carbon contents. $\alpha$ - chymotrypsin enzyme was the only enzyme capable for removing this amorphous material.

Conclusion: Ultrastructure analysis of the affected teeth showed typical form of hypomaturation amelogenins imperfecta. The study also proved that this amorphous material is mainly proteinases in nature.

KEYWORD: Enamel, Scanning Electron Microscope, Energy Dispersive X-Ray Spectroscopy, Amelogenesis imprecate

* Oral Biology Department, Suez Canal University- Ismailia, Egypt

** Basic Dental Science Department- Gulf Medical University-UAE 


\section{INTRODUCTION}

Amelogenesis or enamel formation is a biomineralization process in which specialized epithelial derived cells, ameloblasts, secrete extracellular matrix which later will be mineralized (Smith et al., 2019). Although there are variations in the enamel pattern ultrastructure between different species, the way by which enamel is formed is highly conserved between all species (Kawasaki et al., 2014). Enamel is unique among human biomineralized tissues and the process of its formation (amelogenesis) is very complicated (Pham et al., 2017). For simplicity, amelogenesis can be divided into two main stages; the secretory stage and the maturation stage (Pham et al., 2017). During the secretory stage, ameloblasts, enamel forming cells, differentiate and form distal junctional complexes and start to secrete a proteinrich, partially mineralized, self-organizing matrix) (Smith et al., 2011). This matrix is composed of $50 \%$ (by volume) enamel matrix proteins (mainly Amelogenin [AMELX]; Enamelin [ENAM]; Ameloblastin [AMBN] and Matrix metalloproteases 20 [MMP20]) and 30\% minerals (mainly $\mathrm{Ca}^{2+}$, $\mathrm{PO}_{4}^{2-}$ and $\mathrm{PO}_{4}^{4-}$ ) (Smith et al., 2011). Due to the interactions these different proteins are involved in, both with each (protein-protein interaction) and with the mineral phase of the secreted matrix (protein-mineral interactions); thin, needle-like hydroxyapatite crystals initially develop and then elongate to form the whole thickness of the enamel (Smith et al., 2011).

Any defect that happens during the secretory stage will result in abnormal crystal nucleation and/or elongation of enamel crystals, resulting in enamel hypoplasia (thin enamel) (Ruschel et al., 2016). After formation of an enamel layer of normal thickness, ameloblasts are reduced in size and almost $50 \%$ of their population die (Nanci et al., 1989). The fundamental process of enamel biomineralization occurring during the maturation stages of amelogenesis is deposition of large number of ions (Ca2+, PO42- and PO4-) onto the side of the highly elongated pre-existing enamel crystals (Moradian-Oldak et al., 1996). This leads to growth of these crystals in width and thickness until they about each other to give enamel its distinctive properties. In order to let this, happen, ameloblasts remove nearly all enamel fluid and degraded enamel matrix proteins (Smith et al., 1989). Any defects in removing the enamel matrix proteins or in the deposition of ions will result in failure of enamel crystals to grow in width and thickness, leading to improper enamel mineralization (Simmer et al., 2009).

Amelogenesis imperfecta (AI), is a group of conditions, genomic in origin, which affect the structure and clinical appearance of enamel of all, or nearly all the teeth, and which may be associated with morphologic or biochemical changes elsewhere in the body ( $\mathrm{Ng}$ and Messer, 2009). Clinically, AI is classified according to the clinical phenotype and the mode of inheritance into three main groups; Hypoplastic, Hypocalcified and Hypomaturation types (Ng and Messer, 2009, Aldred et al., 2003). The aim of this work is to investigate the ultrastructure phenotype of permanent and deciduous teeth with generalized enamel defects (Amelogenesis imperfecta).

\section{MATERIALS AND METHODS}

\section{Scanning Electron Microscopy (SEM) Energy Dispersive X-Ray Spectroscopy (EDX)}

Teeth whether deciduous ( $\mathrm{n}=2$ Molars $)$ or permanent ( $\mathrm{n}=2$ premolars) (extracted for clinical reasons), were collected from affected individuals. The teeth were either embedded in an embedding wax if there was enough remaining sound tooth structure or in methyl methacrylate if badly decayed. Teeth were sectioned longitudinally in the bucco-lingual direction using an Accutom-5 cutter with a peripheral diamond cutting disc, cooled with 
minimum water to minimize loss of any organic components within the enamel. One section in the midline was polished plano-parallel with wet 1200 -grade carborundum paper to a thickness of $100-120 \mu \mathrm{m}$, then acid etched for exactly 15 seconds with $35 \%$ phosphoric acid to remove the smear layer. The sections were then washed by $\mathrm{dH} 2 \mathrm{O}$ to stop the acid etching and to remove any debris on the surface (Shore et al., 2002).

The sections were mounted on aluminium stubs and sputter coated with gold. Microstructural analysis was undertaken using a Jeol 35 SEM fitted with the Deben Genie upgrade (Deben Engineering, Debenham, UK). Elemental analysis was performed by energy dispersive X-ray spectroscopy (EDX) using a detector fitted with an ultrathin window and driven by WinEDX 3 software (Thomson Scientific, Carlton, Australia) (Shore et al., 2002).

\section{Extraction of organic material}

In order to determine the amorphous material that sometimes appeared inside the enamel structure was a genuine finding or not and trying also to identify the nature of such amorphous material, a selective removal was carried out using either $\alpha$ chymotrypsin (Sigma-UK) to test if this material was protein or using lipase (Sigma-UK) to determine if it was lipid.

\section{Enzyme digestions}

Three of the sections which were scanned initially from the affected enamel were re-polished using 1200 grinding paper and then washed with water for at least 1 hour. These sections were then etched with $35 \%$ phosphoric acid for 15 seconds. One section was then incubated in a (1-100 w/v) solution of $\alpha$ - chymotrypsin (C5088-2MG -Sigma-UK) and 10mM Tris HCL pH7.7 buffer, while another was incubated in a solution of the same concentration of lipase (Sigma-UK) and 10mM Tris pH7.7 buffer.
The last section was incubated in Tris buffer alone to act as a control. All the sections were then incubated at $37^{\circ} \mathrm{C}$ for 12 hours. Control equivalent sections of normal unaffected enamel were also subjected to this all experimental procedure. After that the sections were washed again with water for at least 1 hour and then dried overnight in a dry air vacuum. The sections were recoated with gold and scanned again across the fracture surface (Shore et al., 2002).

\section{RESULTS}

\section{SEM and EDX}

Typical hypomineralised teeth were observed in the proband of a highly consanguineous family. The affected proband and his cousin were born to unaffected parents, consistent with an autosomal recessive inheritance pattern (it is not part of the selection criteria, it is a results of analysis family pedigree). Teeth from both affected individuals were characterized by marked yellow-brown discoloration in both the primary and secondary dentitions.

Examination of sections of affected teeth from both primary and secondary dentitions gave a consistent pattern on SEM examination. This was characterized by a normal thickness of enamel that demonstrates a prominent amorphous material located in the middle of the enamel but not in the cervical region or towards the outer-most surface (Fig.1). This amorphous material covered approximately half of the prepared enamel surface from tooth surface to DEJ, the underlying enamel structure being obscured to a great extent by this material. Wherever the enamel structure was visible, it revealed loss of normal enamel architecture with irregular and incompletely formed prism structure (Fig.2). The underlying dentine was also covered by this amorphous material with irregular and apparently fewer dentinal tubules (Fig.2). 


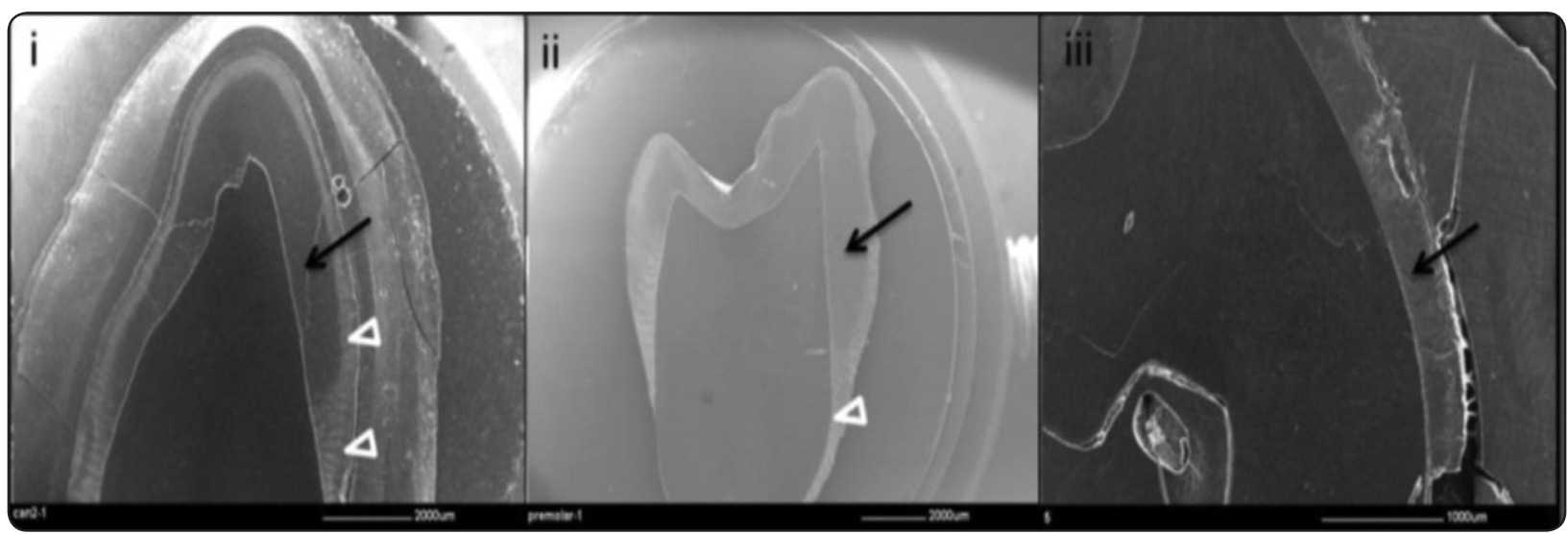

Fig. (1) SEM Examination of Enamel of Permanent and Deciduous Teeth

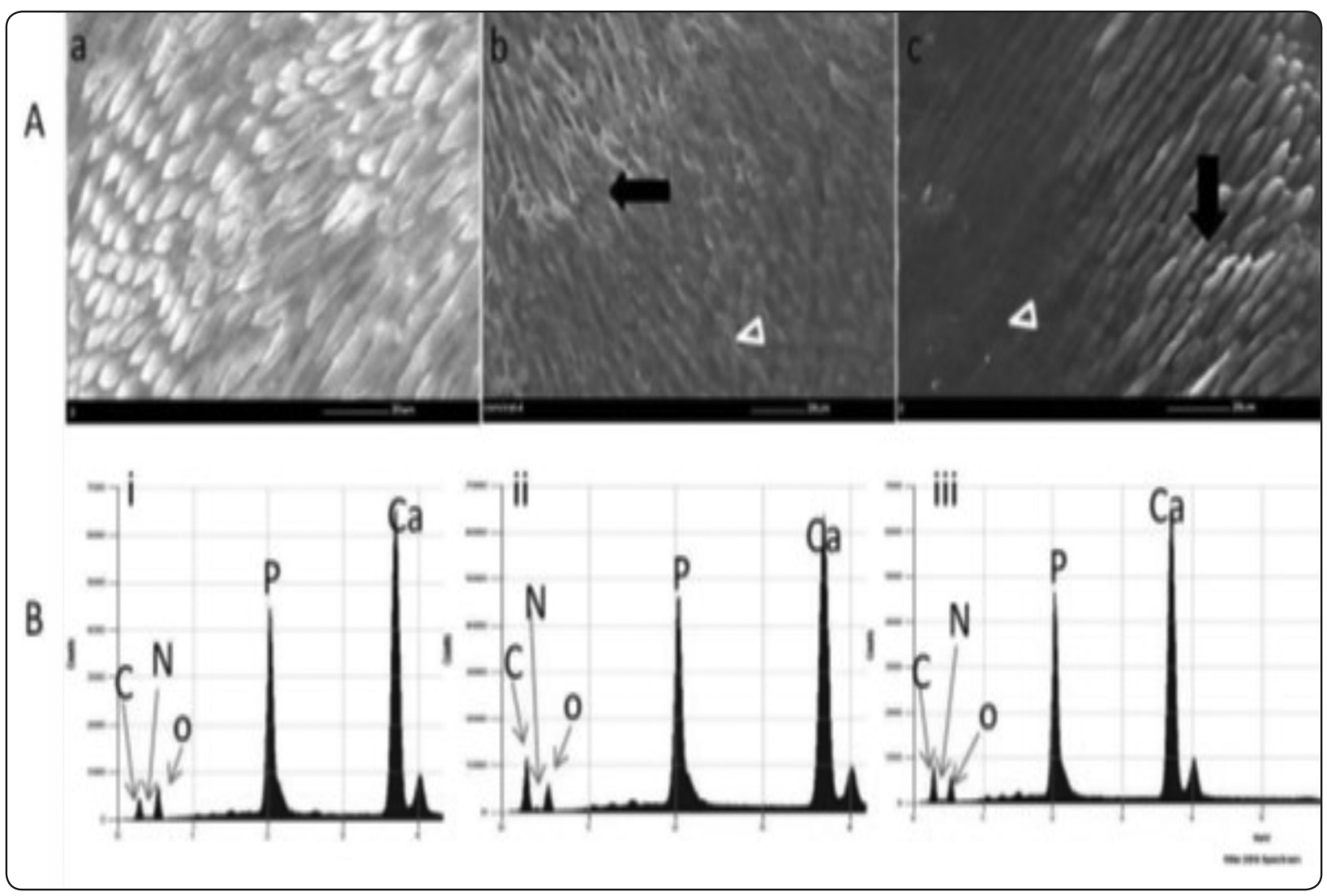

Fig. (2) Ultrastructural analysis of permanent lower second premolar and deciduous lower second molar teeth from affected member and control individual

SEM appearance of control enamel (a) that contrasts with (b) the poorly formed enamel rods of affected permanent lower second premolar or (c) affected deciduous lower second molar. The enamel rods in affected enamel are obscured by the amorphous material (open white arrows), bars $20 \mu \mathrm{m}$. (B) EDX spectra for corresponding SEM pictures; in control enamel (i), the carbon: oxygen $(\mathrm{C}: \mathrm{O})$ ratio is low compared to that in affected enamel (ii and iii). A small nitrogen $(\mathrm{N})$ peak was observed (between the $\mathrm{C}$ and $\mathrm{O}$ peaks) in affected, but not control teeth. Similar peaks are observed in affected and control teeth for calcium $(\mathrm{Ca})$ and phosphate $(\mathrm{P})$. 


\section{Enzyme digestion}

To characterize the biochemical nature of the amorphous material, previously scanned sections with SEM were re-polished and re-etched with $35 \%$ phosphoric acid for 15 seconds and incubated in $\alpha$-chymotrypsin (C5088-2MG -Sigma-UK), lipase (16698-100MG-F -Sigma-UK) or Tris buffer (control). Following incubation of teeth sections in different solutions, $\alpha$-chymotrypsin enzyme was the only one that removed the amorphous material from the enamel structure (Fig.3). The appearances were unaltered after incubation with lipase or Tris buffer alone. Once the amorphous material had been removed, the revealed enamel structure was characterized by highly irregular and incompletely formed enamel rods. Porosity and small cavities were also observed (Fig.3).
Repeating EDX after removal of the amorphous material gave a spectrum indistinguishable from that of control enamel, indicating that the amorphous material was the source of the increase of the carbon (C) and nitrogen (N) peaks (Fig. 3). Examination of sections from deciduous teeth treated in the same way as the permanent tooth sections, gave the same results (Fig.3). This indicates that the effect is the same on both primary and secondary dentitions.

Sections of permanent upper canine (i), lower second premolar (ii) and deciduous lower second molar (iii) from patient VII:2 illustrate a near-normal thickness of enamel with an amorphous material over much of the prepared enamel surface (black arrows). The amorphous material was absent from the cervical and the outer-most aspects of the enamel (open arrows), bar $2000 \mu \mathrm{m}$ (i and ii), and $1000 \mu \mathrm{m}$ (iii).

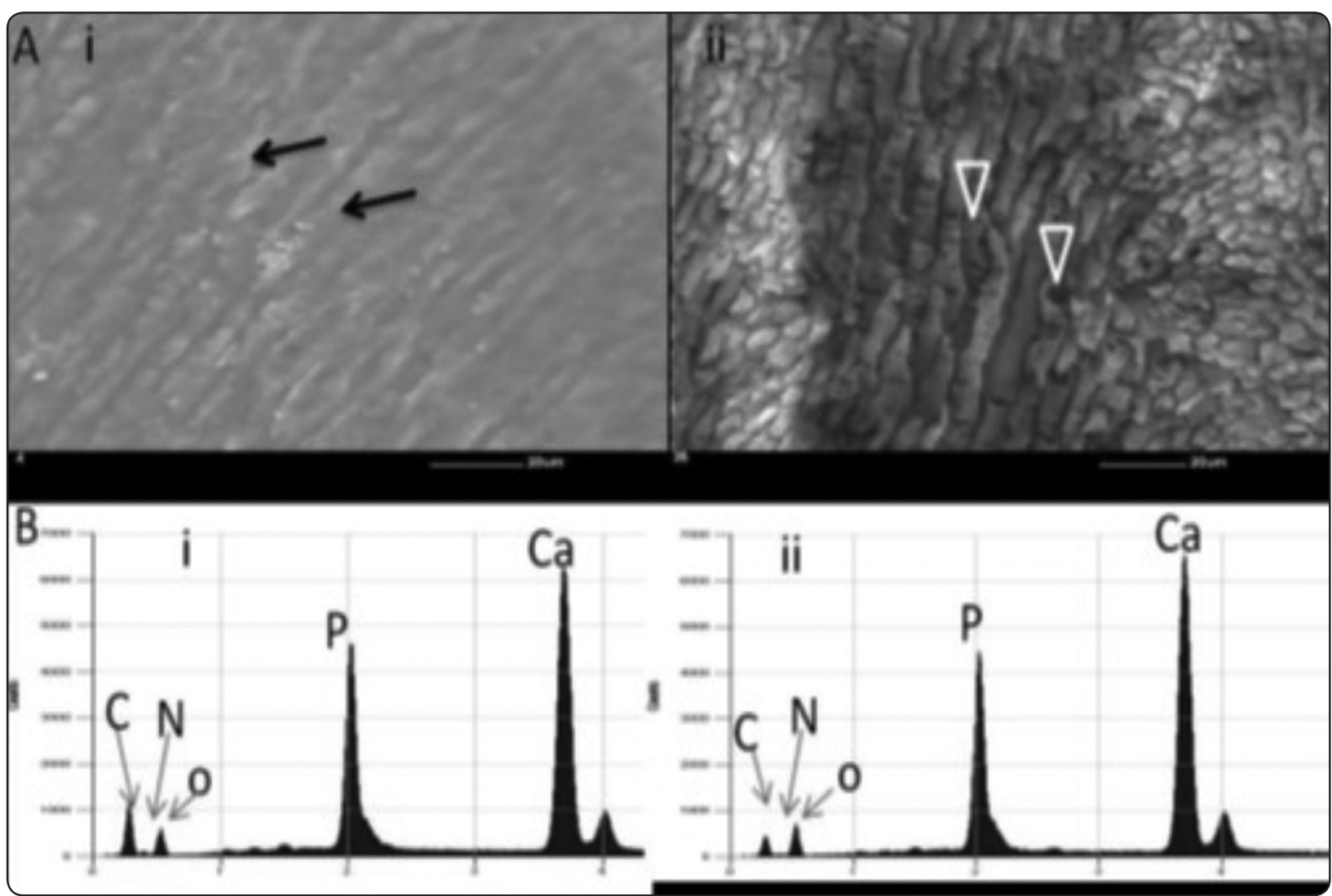

Fig. (3) Ultrastructural examinations of permanent teeth from affected member before (i) and after (ii) incubation with $\alpha$-chymotrypsin.

A) SEM of affected permanent enamel before (i) and after (ii) incubation with $\alpha$-chymotrypsin. (i) SEM of affected enamel illustrates irregular enamel rods (black arrows) partially obscured by the amorphous material. (ii) Enamel rods are more readily observed after removal of the amorphous material by the activity of $\alpha$-chymotrypsin. Enamel rods are poorly formed with multiple porosities (white open arrows). Bar $20 \mu \mathrm{m}$. B) EDX spectra of enamel before (i) and after (ii) treatment with $\alpha$-chymotrypsin; Carbon $(\mathrm{C})$ and oxygen $(\mathrm{O})$ peaks have returned to a ratio consistent with that seen in normal control after incubation of the enamel with the $\alpha$-chymotrypsin 


\section{DISCUSSION}

Amelogenesis or the process of enamel formation is highly genetically controlled which depends on the contributions of many genes. This led to enamel malformation phenotype to be presented as a clinical feature for about 90 inherited conditions (Wright et al., 2015). As it is already known that deciduous teeth start to erupt in the first year and the teeth set is completely erupted by the third year of age, there is an early presentation of dental enamel malformations to clinicians. Because of that, the enamel phenotype is considered and early and may be the only apparent sign at the time of initial diagnosis. Abnormally thin (hypoplastic) enamel is characteristic of an early disturbance during amelogenesis, and soft enamel of normal thickness (hypomaturation) is characteristic of a late disturbance (Witkop, 1988, Wright et al., 2015).

In our study, the clinical data indicate that the affected enamel from the affected patient is of normal thickness and the teeth have normal morphology. This indicates that the type of defect is amelogenesis imperfecta of hypomaturation type and not hypoplastic type according to the classical and common classification of AI (Witkop, 1988, Wright et al., 2015).

The ultrastructure analysis of the affected enamel showed poorly formed enamel rods. In addition, the enamel rods in affected enamel are obscured by the amorphous material. This typical description of enamel rod defects and presence of the amorphous material in the enamel structure has been described with hypomineralized enamel defects in other studies (Shore et al., 2002, El-Sayed et al., 2011, El-Sayed et al., 2010). This supports the idea that this material is a secondary event reflecting failure of ameloblasts to remove degraded proteins as required for enamel crystal maximum maturation.

Increase in the carbon $(\mathrm{C})$ and nitrogen $(\mathrm{N})$ content observed in the EDX spectra from affected enamel indicates that this material is organic in nature. It has been demonstrated in other studies that hypomaturation AI enamel has less mineral and more protein than normal teeth (Wright et al., 1995, Wright et al., 2006, Simmer et al., 2009). The efficacy of $\alpha$-chymotrypsin in removing this material is consistent with high protein content and not lipids which was introduced by Shore et al (Shore et al., 2002).

It has been evident for some time now that the maturation stage ameloblasts undergo cyclic modulations (Smith, 1998, Smith et al., 2016, Nunez et al., 2016, Robinson, 2014). During these modulations, ameloblasts have two forms: ruffleended (RE) ameloblasts, which have tight distal and loose proximal junctional complexes with a striated border toward the enamel surface, and smooth-ended (SE) ameloblasts, which have loose distal and tight proximal junctional complexes and non-striated distal membranes (Smith, 1998, Smith et al., 2016, Nunez et al., 2016, Robinson, 2014). This cyclic modulation is believed to be essential for ameloblast functions, which are important for enamel maturation (Smith, 1998, Smith et al., 2016, Nunez et al., 2016, Robinson, 2014).

During the RE-stage, ameloblasts reabsorb all the degraded proteins and enamel fluids leaving porous enamel (Robinson, 2014). The exact mechanism by which ameloblasts reabsorb the degraded proteins is not well understood. However, it is suggested recently that the degraded enamel matrix proteins bind to the cell surface receptors of ameloblast plasma membrane and this binding enhances endocytosis and pinocytosis of the ameloblast (Pham et al., 2017). The degraded proteins then are translocated from the plasma membrane to the lysosomes where they can be degraded. Due to removal of the degraded proteins and large amount of enamel fluid during the RE-stage leaving porous enamel, different ions $\left(\mathrm{Mg}^{2+}, \mathrm{K}^{+} 202\right.$ and $\left.\mathrm{Na}^{+}\right)$can adsorb to the surface of the growing hydroxyapatite crystals (Fukae et al., 2002, Ryu et al., 1998). So, 
any defects in this stage will result in pours enamel and this explains why in our work, the enamel rod was porous. Also, ameloblasts needs to increase the flux of $\mathrm{Ca}^{2+}$ and $\mathrm{PO}_{4}^{2-}$ into the enamel matrix to enhance crystal growth and disappearing of this porosity. While, SE-ameloblasts are associated with neutralizing the acid released by formation of hydroxyapatite crystals during the RE. This involves the production of bicarbonate (HCO3-) by carbonic anhydrase II (CA2) and its transport into the matrix by anion exchanger II (AE2) (Yin et al., 2015).

\section{REFERENCES}

1. Aldred, M. J., Savarirayan, R. \& Crawford, P. J. 2003. Amelogenesis imperfecta: a classification and catalogue for the 21st century. Oral Dis, 9, 19-23.

2. El-Sayed, W., Shore, R. C., Parry, D. A., Inglehearn, C. F. \& Mighell, A. J. 2010. Ultrastructural analyses of deciduous teeth affected by hypocalcified amelogenesis imperfecta from a family with a novel Y458X FAM83H nonsense mutation. Cells Tissues Organs, 191, 235-9.

3. El-Sayed, W., Shore, R. C., Parry, D. A., Inglehearn, C. F. \& Mighell, A. J. 2011. Hypomaturation amelogenesis imperfecta due to WDR72 mutations: a novel mutation and ultrastructural analyses of deciduous teeth. Cells Tissues Organs, 194, 60-6.

4. FUKAE, M., TANABE, T., NAGANO, T., ANDO, H., YAMAKOSHI, Y., YAMADA, M., SIMMER, J. P. \& OIDA, S. 2002. Odontoblasts enhance the maturation of enamel crystals by secreting EMSP1 at the enamel-dentin junction. J Dent Res, 81, 668-72.

5. KAWASAKI, K., HU, J. C. \& SIMMER, J. P. 2014. Evolution of Klk4 and enamel maturation in eutherians. Biol Chem, 395, 1003-13.

6. Moradian-Oldak, J., Leung, W., Simmer, J. P., ZeichnerDavid, M.\& Fincham, A. G. 1996. Identification of a novel proteinase (ameloprotease-I) responsible for the complete degradation of amelogenin during enamel maturation. Biochem J, 318 ( Pt 3), 1015-21.

7. Nanci, A., Ahluwalia, J. P., Zalzal, S. \& Smith, C. E. 1989. Cytochemical and biochemical characterization of glycoproteins in forming and maturing enamel of the rat incisor. J Histochem Cytochem, 37, 1619-33.
8. NG, F. K. \& Messer, L. B. 2009. Dental management of amelogenesis imperfecta patients: a primer on genotypephenotype correlations. Pediatr Dent, 31, 20-30.

9. Nunez, S. M., Chun, Y. P., Ganss, B., HU, Y., Richardson, A. S., Schmitz, J. E., Fajardo, R., Yang, J., HU, J. C. \& Simmer, J.P. 2016. Maturation stage enamel malformations in Amtn and Klk4 null mice. Matrix Biol, 52-54, 219-233.

10. Pham, C. D., Smith, C. E., Hu, Y., Hu, J. C., Simmer, J. P. \& CHUN, Y. P. 2017. Endocytosis and Enamel Formation. Front Physiol, 8, 529.

11. Robinson, C. 2014. Enamel maturation: a brief background with implications for some enamel dysplasias. Front Physiol, 5, 388.

12. Ruschel, V. C., Araujo, E., Bernardon, J. K. \& Lopes, G. C. 2016. Enamel hypoplasia: challenges of esthetic restorative treatment. Gen Dent, 64, 75-8.

13. Ryu, O. H., HU, C. C. \& Simmer, J. P. 1998. Biochemical characterization of recombinant mouse amelogenins: protein quantitation, proton absorption, and relative affinity for enamel crystals. Connect Tissue Res, 38, 207 14; discussion 241-6.

14. Shore, R. C., Backman, B., Brookes, S. J., Kirkham, J., Wood, S. R. \& Robinson, C. 2002. Inheritance pattern and elemental composition of enamel affected by hypomaturation amelogenesis imperfecta. Connect Tissue Res, 43, 466-71.

15. Simmer, J. P., HU, Y., Lertlam, R., Yamakoshi, Y. \& HU, J . C. 2009. Hypomaturation enamel defects in Klk4 knockout/LacZ knockin mice. J Biol Chem, 284, 19110-21.

16. SMITH, C. E. 1998. Cellular and chemical events during enamel maturation. Crit Rev Oral Biol Med, 9, 128-61.

17. Smith, C. E., HU, Y., HU, J. C. \& Simmer, J. P. 2016. Ultrastructure of early amelogenesis in wild-type, Amelx(-/-), and Enam(-/-) mice: enamel ribbon initiation on dentin mineral and ribbon orientation by ameloblasts. Mol Genet Genomic Med, 4, 662-683.

18. Smith, C. E., HU, Y., HU, J. C. \& Simmer, J. P. 2019. Quantitative analysis of the core 2D arrangement and distribution of enamel rods in cross-sections of mandibular mouse incisors. J Anat, 234, 274-290.

19. Smith, C. E., Hu, Y., Richardson, A. S., Bartlett, J. D., HU, J. C. \& Simmer, J. P. 2011. Relationships between protein and mineral during enamel development in normal and genetically altered mice. Eur J Oral Sci, 119 Suppl 1, 125-35. 
20. Smith, C. E., Pompura, J. R., Borenstein, S., Fazel, A. \& Nanci, A. 1989. Degradation and loss of matrix proteins from developing enamel. Anat Rec, 224, 292-316.

21. Witkop, C. J., JR. 1988. Amelogenesis imperfecta, dentinogenesis imperfecta and dentin dysplasia revisited: problems in classification. J Oral Pathol, 17, 547-53.
22. Wright, J. T., Carrion, I. A. \& Morris, C. 2015. The molecular basis of hereditary enamel defects in humans. J Dent Res, 94, 52-61.

23. Yin, K., Lei, Y., Wen, X., Lacruz, R. S., Soleimani, M., Kurtz, I., Snead, M. L., White, S. N. \& Paine, M. L. 2015. SLC26A Gene Family Participate in pH Regulation during Enamel Maturation. PLoS One, 10, e0144703. 